\title{
MicroRNA-320a Regulates the Osteogenic Differentiation of Human Bone Marrow- Derived Mesenchymal Stem Cells by Targeting HOXA10
}

\author{
Jianhou Huang ${ }^{\mathrm{a}}$ Yake Meng ${ }^{\mathrm{b}}$ Yan Liu ${ }^{\mathrm{b}}$ Yu Chen ${ }^{\mathrm{b}}$ Haisong Yang ${ }^{\mathrm{b}}$ Deyu Chen \\ Jiangang Shi ${ }^{b}$ Yongfei Guo ${ }^{b}$ \\ aDepartment of Orthopedics, The 188th Hospital of PLA, ChaoZhou, GuangDong Province, China, \\ bDepartment of Orthopedics, ChangZheng Hospital, Second Military Medical University, Shanghai, \\ China
}

\section{Key Words}

Bone marrow-derived mesenchymal stem cells • Osteogenic differentiation • HOXA10 • MiR$320 a$

\begin{abstract}
Background/Aims: Human bone marrow-derived mesenchymal stem cells (hMSCs) are a promising cell source for bone engineering owing to their high potential to differentiate into osteoblasts. The bone morphogenetic protein-inducible gene homeobox a10 (HOXA10) is a critical regulator of osteogenesis. The objective of the present study was to identify microRNAs (miRNAs) targeting HOXA10 and examine the effects on the osteogenic differentiation of hMSCs. Methods: Based on in silico analysis, HOXA10-targeting miRNAs were selected and their regulatory roles in osteoblast differentiation were investigated. Results: Six HOXA10-targeting miRNAs were identified by computational analysis, of which miR-320a was selected for further analysis because it was downregulated during osteogenic induction. Overexpression of miR-320a downregulated HOXA10 and significantly inhibited osteogenesis in hMSCs, as determined by the downregulation of the osteogenic markers Runx2, ALP, and OC and the inhibition of ALP activity and matrix mineralization, whereas miR-320a inhibition had the opposite effects. Furthermore, ectopic expression of HOXA10 (not including 3'-UTR) rescued the effects of miR-320a on osteogenic differentiation. Conclusion: These results suggest that miR-320a acts as a critical regulator of osteogenic differentiation of hMSCs by repressing its target HOXA10.
\end{abstract}




\section{Cellular Physiology Cell Physiol Biochem 2016;38:40-48

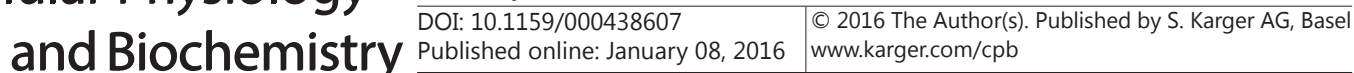 \\ Huang et al.: miR-320a Regulates Osteogenic Differentiation of hMSCs}

\section{Introduction}

Bone marrow-derived stromal (mesenchymal and skeletal) stem cells, also termed human mesenchymal stem cells (hMSCs), are precursors of nonhematopoietic tissues that have attracted attention because of their capacity for self-renewal, differentiation potential and possible use for cell and gene therapy [1]. The differentiation of hMSCs into various mesenchymal tissue lineages including bone, cartilage, fat, tendon, and muscle occurs in response to different environmental stimuli and is regulated by complex pathways [2, 3]. MicroRNAs (miRNAs), which are small (22-nucleotide) noncoding RNAs that regulate gene expression by specific binding to the $3^{\prime}$-untranslated region ( $3^{\prime}$-UTR) of target mRNAs, have recently been implicated in the regulation of hMSC differentiation into various lineages [4-7]. Three miRNAs (miR-96, miR-124 and miR-199a) differentially expressed during mesenchymal lineage commitment of mouse stromal cells were originally identified and shown to be selectively upregulated during osteogenesis, chondrogenesis or adipogenesis [8]. In addition, lineage-specific miRNAs regulate the differentiation of multipotent cells into one phenotype by blocking alternative fates [9]. Many miRNAs have been identified as positive and negative regulators of osteogenic differentiation of hMSCs by modulating the expression of genes involved in bone development, suggesting their potential therapeutic application for the treatment of bone-related diseases [10, 11]. Furthermore, deregulation of miRNAs has been implicated in the pathogenesis of diseases involving bone degeneration, such as osteoporosis [12].

Homeobox a10 (HOXA10), a bone morphogenetic protein (BMP) 2 inducible gene, contributes to osteogenic lineage determination by activating the osteogenic transcription factor Runx2 and modulating osteoblast gene expression [13]. HOXA10 was shown to be a target of miR-135a in breast cancer, endometriosis and ovarian cancer, and to mediate the miR-135a associated invasive phenotype [14]. However, the specific miRNAs targeting HOXA10 and subsequently controlling hMSC commitment to osteoblasts have not yet been fully clarified.

In the present study, we investigated the role of the miRNA mediated modulation of HOXA10 expression in the osteogenic differentiation of hMSCs. Our results showed that miR320 a plays an important role in stem cell fate determination towards an osteoblastic lineage via the regulation of its target HOXA10.

\section{Materials and Methods}

\section{Isolation and culture of hMSCs}

Human bone marrow-derived mesenchymal stem cells (hMSCs) were obtained from posterior iliac crests during routine orthopedic surgery (age: $46 \pm 10$ years; sex: 50/50\% male/female) with written informed consent and agreement. This study was approved by the Medical Ethics Committee of Second Military Medical University. Human hMSCs were isolated using a previously described method [15]. Bone marrow aspirates were harvested using a biopsy needle inserted through the cortical bone. Aspirates were cultured in $\alpha$-MEM (Life technologies; Carlsbad, CA) containing 10\% fetal bovine serum (FBS), $100 \mathrm{U} / \mathrm{ml}$ penicillin and $100 \mathrm{mg} / \mathrm{l}$ streptomycin (Life technologies) at $37^{\circ} \mathrm{C}$ and $5 \% \mathrm{CO}_{2}$. Selection of hMSCs was based on adhesion and proliferation on a plastic substrate. After removal of nonadherent cells, adherent cells were cultured to $90 \%$ confluence and expanded for further experiments.

\section{Osteogenic differentiation of hMSCs}

For osteogenic differentiation of hMSCs, cells were cultured with osteogenic medium (OM) [DMEM containing 10\% FBS, 1\% penicillin/streptomycin, $50 \mu \mathrm{g} / \mathrm{ml} \mathrm{L}$-ascorbic acid, $10 \mathrm{mM} \beta$-glycerophosphate, and $10 \mathrm{nM}$ Dexamethasone (Sigma-Aldrich, Co., St. Louis, USA)]. The osteoblast phenotype was evaluated by alkaline phosphatase (ALP) activity determination as previously described [16]. Briefly, cells were cultured for 10 days and then lysed in lysis buffer composed of Mammalian Protein Extraction Reagent and Protease Inhibitor Cocktail (Cwbiotech, China) according to the manufacturer's instructions. ALP activity 


\section{Cellular Physiology Cell Physiol Biochem 2016;38:40-48 \begin{tabular}{l|l|l}
\hline DOI: 10.1159/000438607 & $\begin{array}{l}\text { C) 2016 The Author(s). Published by S. Karger AG, Basel } \\
\text { www.karger.com/cpb }\end{array}$
\end{tabular}}

Huang et al.: miR-320a Regulates Osteogenic Differentiation of hMSCs

was measured by using $p$-nitrophenylphosphate as a substrate, and the protein content was measured using the bicinchoninic acid (BCA) protein assay kit (Pierce Biotechnology, Rockford, IL, USA). ALP activity was normalized for total protein concentration. Alizarin Red S staining was performed to detect matrix mineralization of hMSCs. After 14 days of OM induction, cells were fixed with ice-cold 70\% ethanol and stained with an Alizarin Red Solution (2\%, pH 4.2, Sigma-Aldrich) for $10 \mathrm{~min}$. Then, cells were washed with distilled water to remove the unbound stain. Images were obtained using a scanner. All experiments were performed in triplicate.

\section{Transfection of Oligonucleotides}

The miRNA mimics, miRNA inhibitors and negative controls of miR-320a were purchased from RiboBio (Guangzhou, China). Transfection of miRNAs was carried out using Lipofectamine 2000 according to the manufacturer's procedure (Invitrogen, Carlsbad, CA).

Total RNA isolation and qRT-PCR Analysis

Total RNA or miRNAs were isolated using the Trizol reagent (Invitrogen) or the mirVana miRNA isolation kit (Ambion, Austin, TX). For analysis of mRNA levels, $1 \mu \mathrm{g}$ of total RNA was reverse transcribed using the SuperScript III first-strand synthesis system supermix (Invitrogen). A SYBR Green PCR kit (TaKaRa, Shiga, Japan) was used to quantify the mRNA levels. $\beta$-actin expression was used as an internal control. For miRNA expression analysis, $1 \mu \mathrm{g}$ of total RNA was converted into cDNA using the TaqMan microRNA reverse transcription kit (Applied Biosystems, Foster City, CA). Expression levels of miRNA were measured using a TaqMan microRNA assay (Applied Biosystems) and normalized to U6 small nucleolar RNA. The data were analyzed using the $2^{-\Delta \Delta C \mathrm{~T}}$ method. All reactions were carried out in triplicate and all experiments were performed three independent times.

\section{Plasmid constructions and transfections}

The HOXA10 expression vector with or without a 300bp miR-320a binding 3 '-UTR fragment was constructed by inserting the HOXA10 CDS into the pcDNA3.1(+) vector (Invitrogen). The $300 \mathrm{bp}$ fragment of the HOXA10 3'-UTR containing the predicted seed sequence for miR-320a was amplified by PCR and cloned into the pRL-TK vector (Promega, Madison, WI). The corresponding mutant constructs were created through site-directed mutagenesis using inverse PCR. The constructs were confirmed by sequencing. Transfection of plasmids was performed using Lipofectamine 2000 (Invitrogen) according to the manufacturer's instructions.

\section{Luciferase assay}

Before transfection, cells were seeded into 96-well plates at 70\% confluence. A mixture of $50 \mathrm{ng}$ p-HOXA10-3'UTR-WT or p-HOXA10-3'UTR-MT, 100 ng miR-320a mimics or miR-CTL, and 10 ng Renilla plasmid were transfected into these cells using Lipofectamine 2000 (Invitrogen) according to the manufacturer's instructions. Luciferase activity was measured in triplicates $48 \mathrm{~h}$ after transfection using the Dual Luciferase Reporter Assay System. Renilla luciferase activity was normalized to firefly luciferase activity.

\section{Western blot analysis}

Proteins were extracted in RIPA buffer [ $25 \mathrm{mM}$ Tris $\cdot \mathrm{HCl}$ (pH 7.6), $150 \mathrm{mM} \mathrm{NaCl}, 1 \%$ Nonidet P-40, $1 \%$ sodium deoxycholate, $0.1 \%$ SDS and protease inhibitor mixture], separated by 10\% SDS-PAGE (Invitrogen), and then transferred onto polyvinylidene difluoride membranes. After blocking in 5\% nonfat milk, the membrane was incubated with primary antibodies against HOXA10 (Santa Cruz, Dallas, TX) or $\beta$-actin (Cell Signaling, Beverly, MA, USA). Then, the membranes were incubated with HRP-conjugated secondary antibody for $1 \mathrm{~h}$ at room temperature and bands were detected using a chemilumminescence reagent.

\section{Statistical analysis}

Results are expressed as mean \pm SD from three independent experiments and differences were compared using Student's two-tailed $t$ test. Probability values were considered statistically significant at $P<0.05$. 


\section{Cellular Physiology Cell Physiol Biochem 2016;38:40-48 \begin{tabular}{l|l|l} 
and Biochemistry & DOI: 10.1159/000438607 & $\begin{array}{l}\text { (c) } 2016 \text { The Author(s). Published by S. Karger AG, Basel } \\
\text { www.karger.com/cpb }\end{array}$
\end{tabular} \\ Huang et al.: miR-320a Regulates Osteogenic Differentiation of hMSCs}

\section{Results}

miR-320a is downregulated during osteogenic differentiation

HOXA10 plays an important role in osteogenic lineage determination of mesenchymal cells [13]. To investigate whether miRNAs could control HOXA10 through binding to the $3^{\prime}$ UTR of HOXA10 mRNA, three miRNA target prediction programs (TargetScan, PicTar and miRanda) were applied to search for HOXA10-targeting miRNAs. Six miRNAs were selected as candidates (Fig. 1A). Analysis of the expression of these miRNAs during osteogenesis by qRT-PCR showed that miR-320a was significantly downregulated in isolated hMSCs under osteogenic induction conditions (OM) compared with general culture conditions (GM) (Fig. 1B). By contrast, the levels of HOXA10 mRNA and protein expression were significantly upregulated when cells were cultured with OM compared to GM (Fig. 1C, D). Collectively, these results revealed that HOXA10 and miR-320a expressions were inversely correlated during osteogenesis of hMSCs, suggesting that miR-320a may be a regulator of HOXA10.

\section{miR-320a acts as an attenuator of HOXA10 expression}

To establish a potential link between the expression levels of HOXA10 and miR-320a during osteogenesis, miR-320a was overexpressed or silenced by transfection of hMSCs with miR-320a mimics or anti-miR-320a oligonucleotides and miR-320a expression was assessed by qRT-PCR (Fig. 2A). Subsequently, analysis of HOXA10 expression showed that miR-320a overexpression significantly downregulated HOXA10 at both mRNA and protein levels, whereas inhibition of miR-320a had the opposite effect (Fig. 2B, C), suggesting that miR-320a targeted the HOXA10 mRNA to suppress its protein expression.

To further confirm the regulation of HOXA10 expression by miR-320a, mutations were introduced into the putative miR-320a binding sites in the HOXA10 $3^{\prime}$-UTR (Fig. 2D), and luciferase reporter constructs generated with the wild-type (WT) and mutant (MT) 3'-UTRs of HOXA10 were co-transfected into hMSCs with miR-320a mimics or miR-control (miRCTL). The results of luciferase assays showed that miR-320a overexpression significantly decreased the luciferase activity of the WT but not that of the MT HOXA10 3'-UTR compared to that of miR-CTL (Fig. 2E), confirming that miR-320a directly targets and downregulates HOXA10 in hMSCs.

A

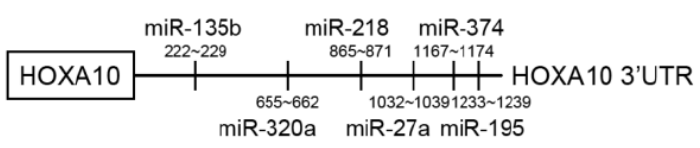

B

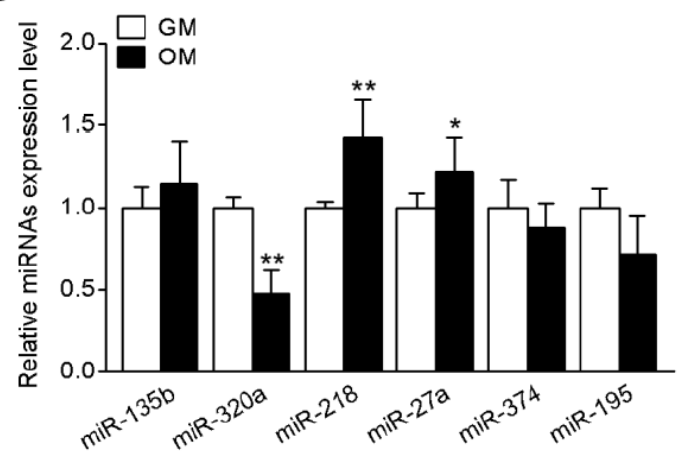

D

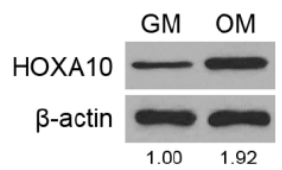

C

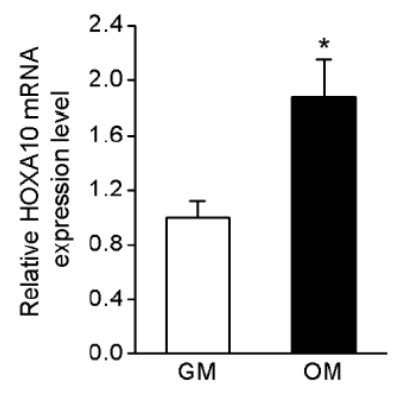

Fig. 1. miR-320a was downregulated during osteoblast differentiation of hMSCs. (A) Schematic representation of the six miRNAs predicted to target the HOXA10-3'-UTR. (B) hMSCs were cultured in osteogenic medium (OM) for 3 days. qRT-PCR analysis of the predicted miRNAs. (C, D) The expression levels of HOXA10 were determined by qRT-PCR and western blotting. Data represent the mean \pm S.D, $\mathrm{n}=3$. $* P<0.05,{ }^{* *} P<0.01$. 


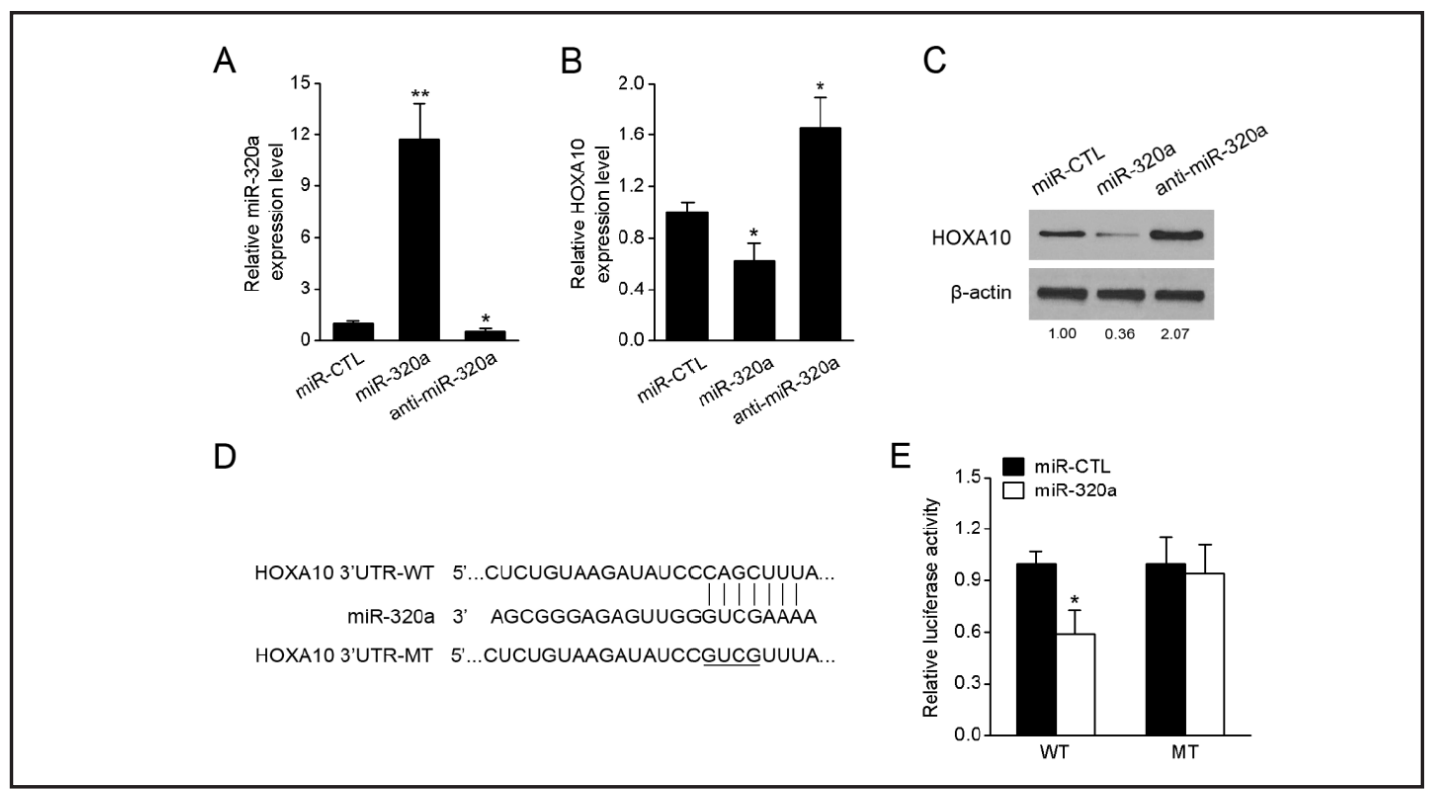

Fig. 2. miR-320a directly targets HOXA10 mRNA. (A, B) hMSCs were transfected with miR-320a mimics or inhibitor and the mRNA expression of miR-320a (A) and HOXA10 (B) were determined by qRT-PCR, and HOXA10 protein levels were determined by western blotting (C). (D) Schematic showing the miR-320a complementary sequence within the HOXA10 3'-UTR and a mutant sequence inserted downstream of the luciferase construct, pRL-TK. (E) Renilla luciferase activity was measured in hMSCs co-transfected with p-HOXA10-3'UTR-WT (WT) or p-HOXA10-3'UTR-MT (MT) and miR-320a mimics or miR-CTL and normalized to firefly luciferase activity. Data represent the mean \pm S.D, $n=3$. ${ }^{*} P<0.05$, ${ }^{* *} P<0.01$.

miR-320a inhibits osteoblast differentiation of hMSCS

Because miR-320a has an inhibitory effect on HOXA10 expression, we determined whether miR-320a regulates osteoblast differentiation of hMSCs. The expression levels of miR-320a were first examined at different time points after incubation of hMSCs in osteogenic induction medium. Expression of miR-320a decreased following osteogenic differentiation, and reached a minimum on day 6 (Fig. 3A). For functional evaluation of miR-320a, hMSCs were transfected with miR-CTL, miR-320a mimics or anti-miR-320a and induced with OM for 7 days. The results showed that miR-320a overexpression significantly downregulated osteoblastic differentiation, which was indicated by lower expression of the osteoblast marker genes Runx2, ALP and osteocalcin (OC) (Fig. 3B), decreased ALP activity, and reduced matrix mineralization visualized by Alizarin red staining (Fig. 3C, D) compared with cells transfected with miR-CTL. By contrast, osteoblast marker gene expression, ALP activity, and matrix mineralization were enhanced in anti- miR-320a-treated hMSCs compared to miRCTL-treated cells. Accordingly, Runx2 and HOXA10 levels were decreased when miR-320a was overexpressed and increased in the absence of miR-320a (Fig. 3E). Taken together, our results suggest that miR-320a is a negative regulator of osteoblast differentiation of hMSCs.

miR-320a regulates osteogenic differentiation of hMSCs via HOXA10

To further explore the role of miR-320a in the osteogenic differentiation of hMSCs, cells were transfected with miR-320a mimics or miR-CTL along with HOXA10 plasmid lacking $3^{\prime}$ UTR or containing wild-type $3^{\prime}$-UTR. qRT-PCR analysis of osteoblast marker genes indicated that ectopic expression of HOXA10 (not including 3'-UTR ) upregulated Runx2, ALP and OC, and reversed the downregulation of these markers of osteogenesis induced by miR-320a overexpression (Fig. 4A). Assessment of ALP activity and Alizarin red staining showed similar results, with HOXA10 restoring ALP activity and matrix mineralization inhibited by miR-320a overexpression (Fig. 4B, C). However, co-transfection of miR-320a mimics and 


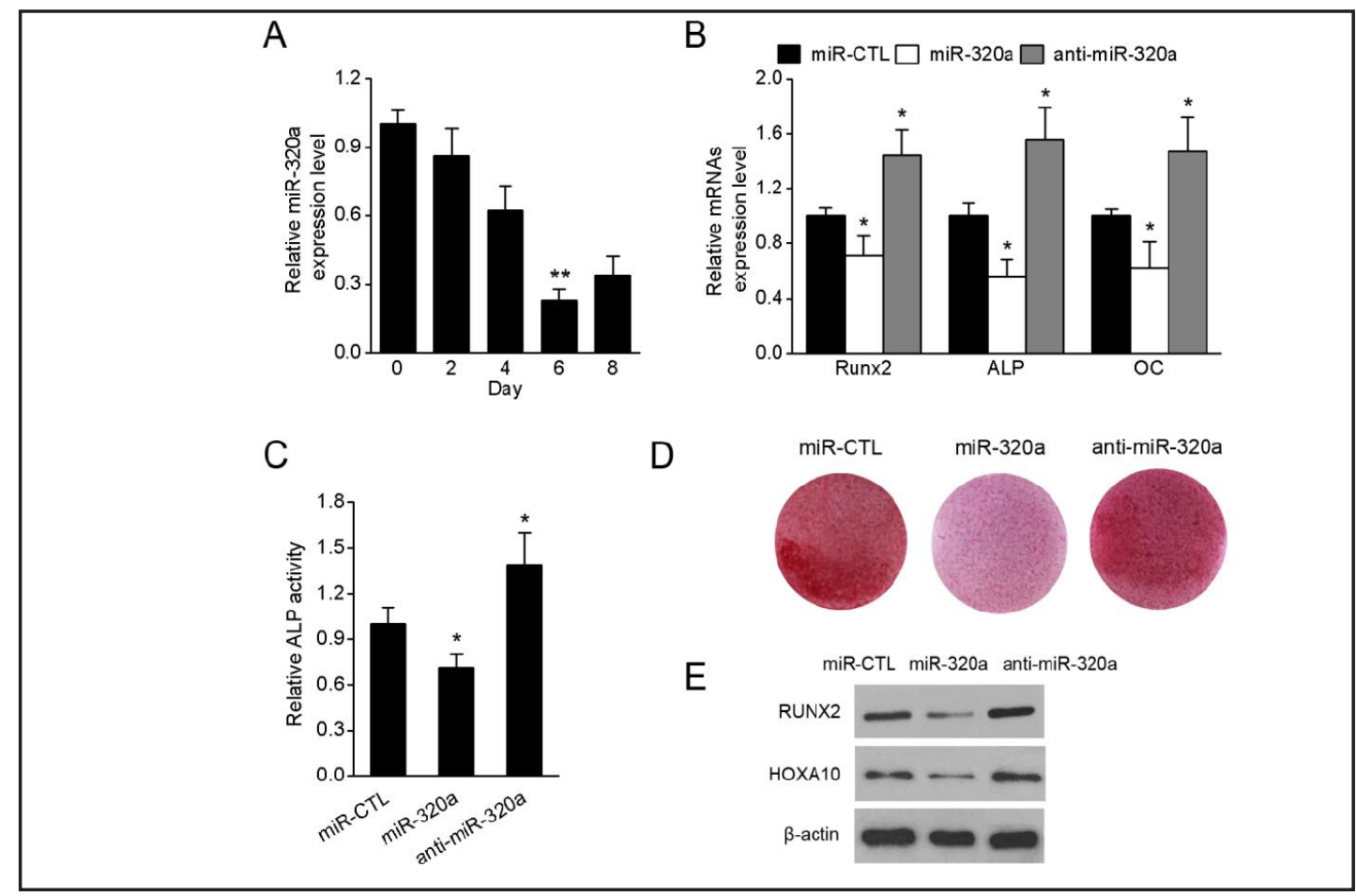

Fig. 3. miR-320a inhibits osteoblast differentiation of hMSCs. (A) qRT-PCR analysis of miR-320a expression during osteogenic induction. (B) hMSCs transfected with miR-CTL, miR-320a mimics or anti-miR-320a were induced with OM for 7 days. Osteoblast differentiation was evaluated with qRT-PCR analysis of osteoblast marker genes (RUNX2, ALP, and OC). (C) ALP activity was measured after culturing in OM for 10 days. (D) Alizarin Red staining was performed after culturing in OM for 14 days. (E) Levels of Runx2 and HOXA10 were analyzed by Western blot at Day 7. Data represent the mean \pm S.D, $\mathrm{n}=3$. $^{*} P<0.05, * * P<0.01$.

HOXA10 containing the 3'UTR sequence didn't abrogate the effects of miR-320a mimics (Fig. 4D). These results suggest that miR-320a inhibits the osteogenic differentiation of hMSCs by downregulating its target HOXA10.

\section{Discussion}

Controlling the osteogenic differentiation of MSCs has many potential therapeutic applications in tissue engineering and regenerative medicine $[17,18]$. Non-coding RNAs, and particularly miRNAs, have been shown to play an important role in the regulation of the osteogenic lineage commitment by modulating transcription factors and signaling pathways involved in bone formation and bone homeostasis [19]. In the present study, we showed that miR-320a plays a pivotal role in the osteogenic differentiation of hMSCs via a mechanism involving the regulation of its target HOXA10.

HOXA10 is essential for normal skeletal development in mammals [20, 21]. HOXA10 inactivation leads to aberrant development of the forelimbs and hind limbs of mice [22, 23]. HOXA10 can regulate the osteogenic factor Runx 2 and its target genes, and regulate osteoblast genes in a manner independent of Runx2, suggesting that HOXA10 functions both during the induction of osteogenesis and in osteoblast differentiation and maturation [13]. The miRNA-mediated regulation of HOXA10 has been demonstrated in several malignancies [24], in particular its association with miR-135 in breast cancer, endometriosis and ovarian cancer [14]. Recently, miR-705 and miR-3077-5p were found to be significantly enhanced in bone marrow MSCs from patients with osteoporosis. Both miR-705 and miR-3077-5p act as inhibitors of MSC osteoblast differentiation by targeting HOXA10 [25]. 
Fig. 4. miR-320a can regulate osteogenic differentiation of hMSCs via HOXA10. (A) hMSCs were cotransfected with miR-320a or miR-CTL and HOXA10 plasmid lacking 3 '-UTR, induced to osteogenic differentiation for 7 days, and then subjected to qRT-PCR analysis of osteoblast marker genes. (B) ALP activity was measured after culturing in OM for 10 days. (C) Alizarin Red staining was performed after culturing in OM for 14 days. (D) qRT-PCR analysis of osteoblast marker genes in hMSCs cotransfected with miR-320a mimics and HOXA10 containing the $3^{\prime}$-UTR. Data represent the mean \pm S.D, $\mathrm{n}=$ 3. ${ }^{*} P<0.05$ compared with the control group, ${ }^{\# P}$ $<0.05$ compared with the miR-320a group.

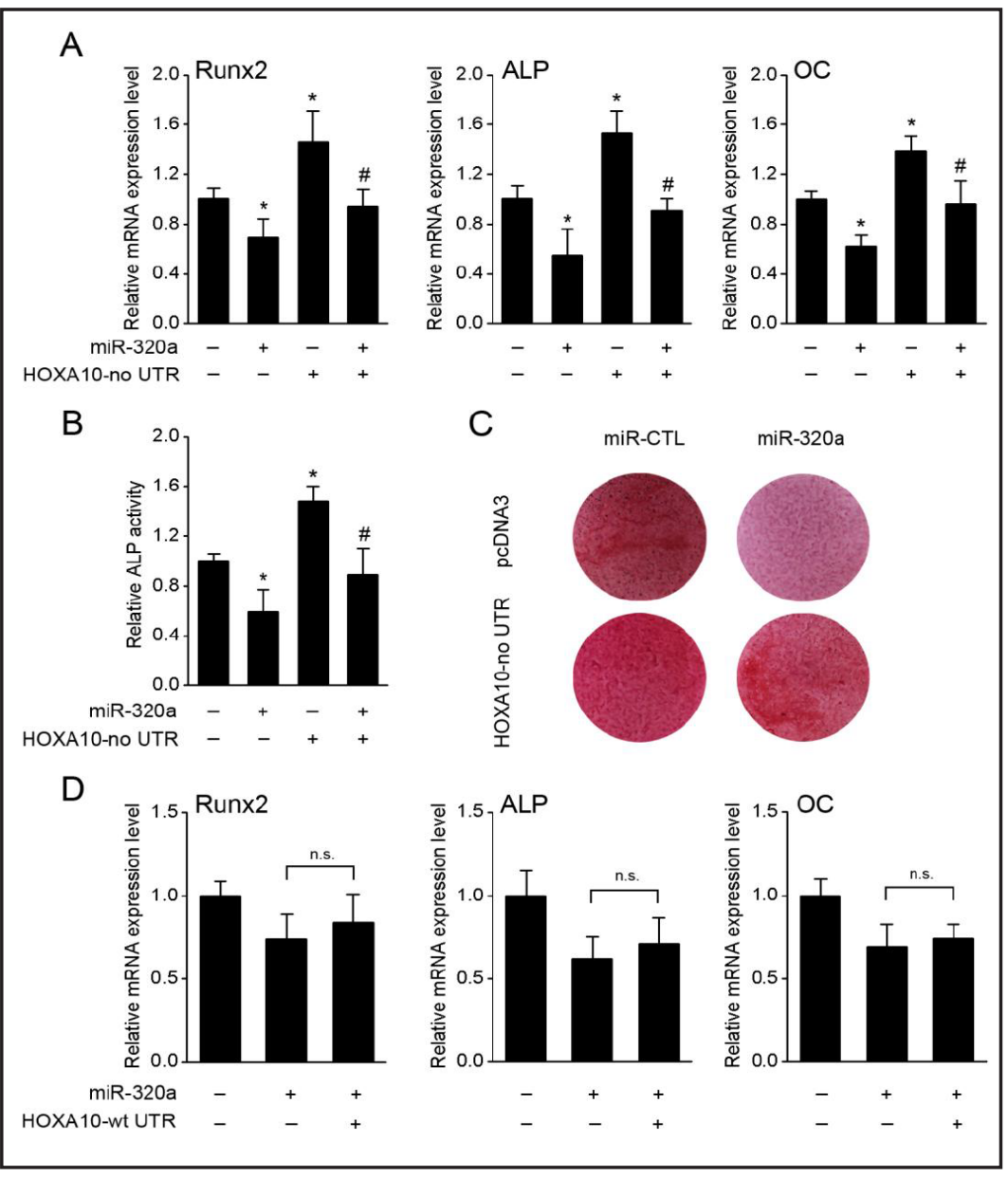

miR-320a was previously shown to be involved in the development and invasiveness of several malignancies including colorectal cancer, nasopharyngeal carcinoma, hepatocellular carcinoma and breast cancer [26-30]. However, its role in MSC differentiation or osteogenic lineage determination has not been analyzed in detail, although it was identified among several miRNAs showing differential expression in association with osteogenic differentiation of human ligament fibroblasts [31].

In this study, we identified a novel function of miR-320a concerning the regulation of HOXA10-mediated osteogenesis. miR-320a was downregulated during the induction of osteogenic differentiation of hMSCs. miR-320a overexpression significantly reduced osteoblastic differentiation, whereas miR-320a inhibition had the opposite effects. It is reported that miR-320 family members are upregulated during adipocyte differentiation of hMSCs, and overexpression of miR-320c promotes adipocytic differentiation of hMSCs [32]. This study together with our findings showing that miR-320a inhibits osteogenesis supports a role for miR-320 in cell lineage determination by promoting the adipogenic and inhibiting the osteogenic phenotype.

The downregulation of miR-320a during osteogenesis occurred concomitant with the upregulation of HOXA10 in hMSCs in the present study. Bioinformatics analysis revealed that the HOXA10 $3^{\prime}$-UTR harbors one potential binding site for miR-320a, and luciferase reporter assays confirmed that HOXA10 is a direct target of miR-320a. Furthermore, our results consistently showed that the levels of HOXA10 mRNA and protein were changed by miR-320a overexpression or inhibition. In addition, HOXA10 overexpression reversed the effects of miR-320a on osteogenesis. Our results strongly support previous reports showing that HOXA10 can induce osteoblast differentiation $[13,25]$, and also suggest that HOXA10 expression might be regulated by miR-320a in osteogenesis.

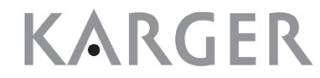




\section{Cellular Physiology Cell Physiol Biochem 2016;38:40-48 \begin{tabular}{l|l|l|}
\hline DOI: 10.1159/000438607 & $\begin{array}{l}\text { C) 2016 The Author(s). Published by S. Karger AG, Basel } \\
\text { www.karger.com/cpb }\end{array}$
\end{tabular} \\ Huang et al.: miR-320a Regulates Osteogenic Differentiation of hMSCs}

In conclusion, we describe a regulatory axis consisting of miR-320a and its target HOXA10 and elucidate the mechanism by which miR-320 inhibits the differentiation of hMSCs into an osteogenic lineage through the downregulation of HOXA10. The present study provides candidate targets for the control of MSC osteogenesis with potential therapeutic application in the treatment of bone diseases.

\section{Acknowledgments}

This work was supported by the National Natural Science Foundation of China (31100988).

\section{Disclosure Statement}

The authors declare that they have no conflict of interest.

\section{References}

1 Colter DC, Class R, DiGirolamo CM, Prockop DJ: Rapid expansion of recycling stem cells in cultures of plastic-adherent cells from human bone marrow. Proc Natl Acad Sci USA 2000;97:3213-3218.

2 Pittenger MF, Mackay AM, Beck SC, Jaiswal RK, Douglas R, Mosca JD, Moorman MA, Simonetti DW, Craig S, Marshak DR: Multilineage potential of adult human mesenchymal stem cells. Science 1999;284:143-147.

3 Zhang H, Li L, Dong Q Wang Y, Feng Q Ou X, Zhou P, He T, Luo J: Activation of pka/creb signaling is involved in bmp9-induced osteogenic differentiation of mesenchymal stem cells. Cell Physiol Biochem 2015;37:548562 .

4 Bartel DP: Micrornas: Target recognition and regulatory functions. Cell 2009;136:215-233.

5 Eskildsen T, Taipaleenmaki H, Stenvang J, Abdallah BM, Ditzel N, Nossent AY, Bak M, Kauppinen S, Kassem M: Microrna-138 regulates osteogenic differentiation of human stromal (mesenchymal) stem cells in vivo. Proc Natl Acad Sci USA 2011;108:6139-6144.

6 Li E, Zhang J, Yuan T, Ma B: Mir-143 suppresses osteogenic differentiation by targeting osterix. Mol Cell Biochem 2014;390:69-74.

7 Gong Y, Xu F, Zhang L, Qian Y, Chen J, Huang H, Yu Y: Microrna expression signature for satb2-induced osteogenic differentiation in bone marrow stromal cells. Mol Cell Biochem 2014;387:227-239.

8 Laine SK, Alm JJ, Virtanen SP, Aro HT, Laitala-Leinonen TK: Micrornas mir-96, mir-124, and mir-199a regulate gene expression in human bone marrow-derived mesenchymal stem cells. J Cell Biochem 2012;113:2687-2695.

9 Suomi S, Taipaleenmaki H, Seppanen A, Ripatti T, Vaananen K, Hentunen T, Saamanen AM, Laitala-Leinonen T: Micrornas regulate osteogenesis and chondrogenesis of mouse bone marrow stromal cells. Gene Regul Syst Bio 2008;2:177-191.

10 Guo L, Zhao RC, Wu Y: The role of micrornas in self-renewal and differentiation of mesenchymal stem cells. Exp Hematol 2011;39:608-616.

11 Taipaleenmaki H, Bjerre Hokland L, Chen L, Kauppinen S, Kassem M: Mechanisms in endocrinology: Micrornas: Targets for enhancing osteoblast differentiation and bone formation. Eur J Endocrinol 2012;166:359371.

12 van Wijnen AJ, van de Peppel J, van Leeuwen JP, Lian JB, Stein GS, Westendorf JJ, Oursler MJ, Im HJ, Taipaleenmaki H, Hesse E, Riester S, Kakar S: Microrna functions in osteogenesis and dysfunctions in osteoporosis. Curr Osteoporos Rep 2013;11:72-82.

13 Hassan MQ Tare R, Lee SH, Mandeville M, Weiner B, Montecino M, van Wijnen AJ, Stein JL, Stein GS, Lian JB: Hoxa10 controls osteoblastogenesis by directly activating bone regulatory and phenotypic genes. Mol Cell Biol 2007;27:3337-3352.

14 Chen Y, Zhang J, Wang H, Zhao J, Xu C, Du Y, Luo X, Zheng F, Liu R, Zhang H, Ma D: Mirna-135a promotes breast cancer cell migration and invasion by targeting hoxa10. BMC Cancer 2012;12:111. 


\section{Cellular Physiology Cell Physiol Biochem 2016;38:40-48 \begin{tabular}{ll|l} 
DOI: 10.1159/000438607 & $\begin{array}{l}\text { O 2016 The Author(s). Published by S. Karger AG, Basel } \\
\text { www.karger.com/cpb }\end{array}$
\end{tabular} \\ Huang et al.: miR-320a Regulates Osteogenic Differentiation of hMSCs}

15 Mauney JR, Jaquiery C, Volloch V, Heberer M, Martin I, Kaplan DL: In vitro and in vivo evaluation of differentially demineralized cancellous bone scaffolds combined with human bone marrow stromal cells for tissue engineering. Biomaterials 2005;26:3173-3185.

16 Onishi M, Fujita Y, Yoshikawa H, Yamashita T: Inhibition of rac1 promotes bmp-2-induced osteoblastic differentiation. Cell Death Dis 2013;4:e698.

17 Jeong BC, Kang IH, Hwang YC, Kim SH, Koh JT: Microrna-194 reciprocally stimulates osteogenesis and inhibits adipogenesis via regulating coup-tfii expression. Cell Death Dis 2014;5:e1532.

18 Chen Y, Chen L, Yin Q, Gao H, Dong P, Zhang X, Kang J: Reciprocal interferences of tnf-alpha and wnt1/ beta-catenin signaling axes shift bone marrow-derived stem cells towards osteoblast lineage after ethanol exposure. Cell Physiol Biochem 2013;32:755-765.

19 Qiao W, Chen L, Zhang M: Microrna-205 regulates the calcification and osteoblastic differentiation of vascular smooth muscle cells. Cell Physiol Biochem 2014;33:1945-1953.

20 Wahba GM, Hostikka SL, Carpenter EM: The paralogous hox genes hoxa10 and hoxd10 interact to pattern the mouse hindlimb peripheral nervous system and skeleton. Dev Biol 2001;231:87-102.

21 Carapuco M, Novoa A, Bobola N, Mallo M: Hox genes specify vertebral types in the presomitic mesoderm. Genes Dev 2005;19:2116-2121.

22 Goodman FR: Limb malformations and the human hox genes. Am J Med Genet 2002;112:256-265.

23 Wellik DM, Capecchi MR: Hox10 and hox11 genes are required to globally pattern the mammalian skeleton. Science 2003;301:363-367.

24 Liborio-Kimura TN, Jung HM, Chan EK: Mir-494 represses hoxa10 expression and inhibits cell proliferation in oral cancer. Oral Oncol 2015;51:151-157.

25 Liao L, Yang X, Su X, Hu C, Zhu X, Yang N, Chen X, Shi S, Jin Y: Redundant mir-3077-5p and mir-705 mediate the shift of mesenchymal stem cell lineage commitment to adipocyte in osteoporosis bone marrow. Cell Death Dis 2013;4:e600.

26 Zhang Y, He X, Liu Y, Ye Y, Zhang H, He P, Zhang Q Dong L, Dong J: Microrna-320a inhibits tumor invasion by targeting neuropilin 1 and is associated with liver metastasis in colorectal cancer. Oncol Rep 2012;27:685694.

27 Zhao H, Dong T, Zhou H, Wang L, Huang A, Feng B, Quan Y, Jin R, Zhang W, Sun J, Zhang D, Zheng M: Mir320a suppresses colorectal cancer progression by targeting rac1. Carcinogenesis 2014;35:886-895.

28 Qi X, Li J, Zhou C, Lv C, Tian M: Microrna-320a inhibits cell proliferation, migration and invasion by targeting bmi-1 in nasopharyngeal carcinoma. FEBS Lett 2014;588:3732-3738.

29 He DX, Gu XT, Jiang L, Jin J, Ma X: A methylation-based regulatory network for microrna 320a in chemoresistant breast cancer. Mol Pharmacol 2014;86:536-547.

30 Yang H, Yu J, Wang L, Ding D, Zhang L, Chu C, Chen Q, Xu Z, Zou Q, Liu X: Mir-320a is an independent prognostic biomarker for invasive breast cancer. Oncol Lett 2014;8:1043-1050.

31 Yu F, Cui Y, Zhou X, Zhang X, Han J: Osteogenic differentiation of human ligament fibroblasts induced by conditioned medium of osteoclast-like cells. Biosci Trends 2011;5:46-51.

32 Hamam D, Ali D, Vishnubalaji R, Hamam R, Al-Nbaheen M, Chen L, Kassem M, Aldahmash A, Alajez NM: Microrna-320/runx2 axis regulates adipocytic differentiation of human mesenchymal (skeletal) stem cells. Cell Death Dis 2014;5:e1499. 